\title{
The Efficacy of Meniscal Treatment Associated with Lateral Tibial Plateau Fractures
}

\author{
Hyun-Joo Park, MD, Hui-Du Lee, MD, and Jin Ho Cho, MD \\ Department of Orthopaedic Surgery, Inje University Ilsan Paik Hospital, Goyang, Korea
}

Purpose: The purpose of this study was to examine the incidence of lateral meniscal tears associated with lateral tibial plateau fractures and report the clinical outcomes of meniscal treatment with internal fixation of fractures.

Materials and Methods: All lateral tibial plateau fractures (Schatzker types II and III) in skeletally mature patients treated operatively at our institution between January 2010 and February 2016 were included. All patients underwent open reduction and internal fixation using a buttress plate or cancellous screws. All meniscal tears were initially considered for repair using an all-inside technique.

Results: The incidence of lateral meniscal tears with lateral tibial plateau fractures was 64\%. Ten patients underwent meniscal repair. In second-look arthroscopy, normal healing was observed in all of the repaired lateral menisci. At the last follow-up, none of the 10 patients had clinical symptoms related to meniscal injuries. One of the 4 patients who had not undergone meniscal treatment although a lateral tear was suspected based on magnetic resonance imaging achieved stable bony union; however, due to the complaint of persisting knee pain, lateral meniscectomy was performed.

Conclusions: Treatment of meniscal lesions associated with lateral tibial plateau fractures showed good clinical and second-look arthroscopic results. Therefore, we believe that recognition and treatment of a meniscal injury at the time of surgical fixation can improve clinical outcome.

\section{Keywords: Knee, Lateral tibial plateau, Fracture, Lateral meniscus, Repair}

\section{Introduction}

Tibial plateau fractures account for $1 \%$ of all fractures and occur relatively frequently in the knee concomitantly with injuries to the meniscus and ligaments ${ }^{1)}$.

In particular, the frequency of lateral tibial plateau fractures that cause metaphyseal split or depression of the articular surface is higher $(55 \%-70 \%)$ than medial and bilateral tibial plateau fractures $(10 \%-23 \%$ and $11 \%-31 \%$, respectively). It has been reported that lateral tibial plateau fractures are significantly associated with lateral meniscal pathology (Schatzker type II, 45\%; type III,

Received May 17, 2016; Revised (1st) August 29, 2016;

(2nd) September 20, 2016; Accepted October 11, 2016

Correspondence to: Jin Ho Cho, MD

Department of Orthopaedic Surgery, Inje University Ilsan Paik Hospital, 170 Juhwa-ro, Ilsanseo-gu, Goyang 10380, Korea

Tel: +82-31-910-9733, Fax: +82-31-910-7967

E-mail:osd07@paik.ac.kr

This is an Open Access article distributed under the terms of the Creative Commons Attribution Non-Commercial License (http://creativecommons.org/licenses/by-nc/4.0/) which permits unrestricted non-commercial use, distribution, and reproduction in any medium, provided the original work is properly cited.
$18 \%)^{2)}$.

Therefore, identification and treatment of the concomitant lesions in the lateral meniscus and ligaments are expected to affect the clinical outcome of the treatment of lateral tibial plateau fractures ${ }^{3)}$.

However, there are insufficient treatment guidelines for lateral meniscal tears associated with tibial plateau fractures due to the lack of follow-up data on meniscal repair in such cases.

The purpose of this retrospective study was to assess the frequency of lateral meniscal tears in patients diagnosed with lateral tibial plateau fractures and evaluate the clinical outcome of meniscal repair.

\section{Materials and Methods}

\section{Patient Group}

We retrospectively reviewed 25 skeletally mature patients who were treated operatively under the diagnosis of lateral tibial plateau fractures at our institution between January 1, 2010 and February 28, 2016. The inclusion criteria were Schatzker type II or III and Arbeitsgemeinschaft für Osteosynthesefragen/Ameri- 

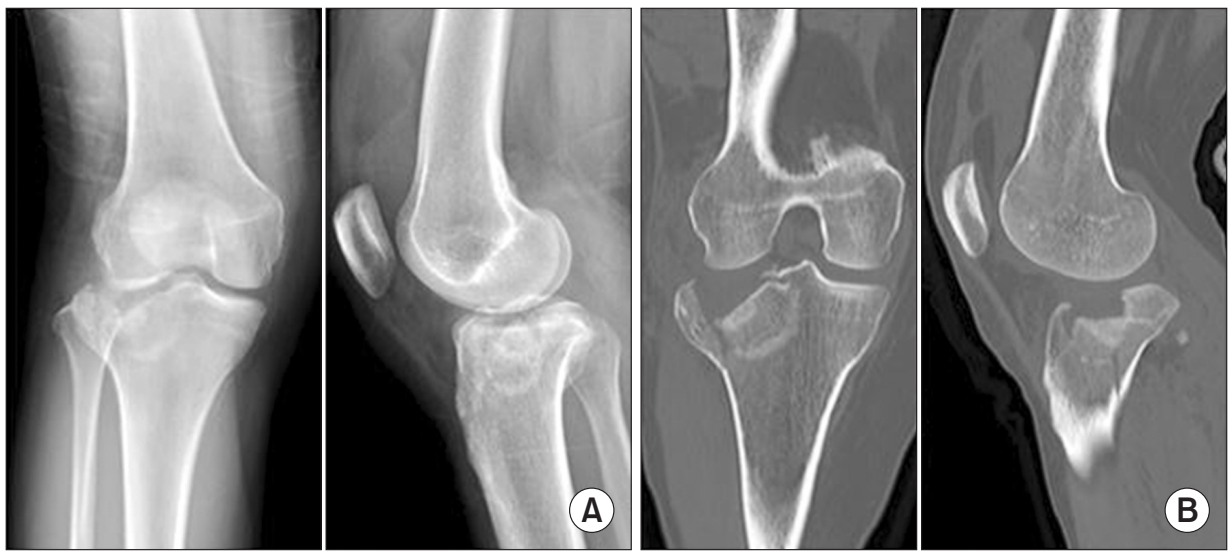

Fig. 1. (A) Anteroposterior and lateral radiographs demonstrating Schatzker II tiabal plateau fracture of the right knee. (B) Coronal and Sagittal computed tomography of the same patient.

can Orthopaedic Trauma Association type 41-B fractures (Fig. 1). Surgical treatment was not considered necessary for Schatzker type I fractures, and thus were excluded from the study.

Of the 25 patients, 17 were male and 8 were female. Their mean age was 49 years (range, 22 to 68 years).

Fracture patterns were classified into Schatzker type II in 15 patients and Schatzker type III in 10 patients. The cause of injury was a traffic accident in 19, a slip down in 3, a sports injury (skiing/football) in 2, and a fall from a height in 1 .

All patients underwent preoperative magnetic resonance imaging (MRI) for identification of an intraarticular injury accompanying the fracture. Lateral meniscal tears were observed in 16 of the 25 patients, medial collateral ligament injuries in 6 , anterior cruciate ligament (ACL) avulsion fractures in 4, posterior cruciate ligament (PCL) injuries in 4 , and a medial meniscus tear in 1 patient (Table 1).

\section{Surgical Technique}

The mean interval from injury to surgery was 3.5 days (range, 0 to 7 days). Under general or spinal anesthesia, the patient was placed in the supine position, and draping was done with a tourniquet applied on the thigh. Prior to fracture reduction, arthroscopic examination was performed to inspect for lesions in the joint. Then, a $10 \mathrm{~cm}$ longitudinal skin incision was made on the anterolateral aspect of the knee to expose the joint and reduce the fracture. In all knees, internal fixation was performed with a buttress plate (TomoFix Lateral High Tibial Plate; DePuy Synthes, West Chester, PA, USA) or cancellous screws.

Reduction of the articular surface was confirmed in two different stages. During reduction of the fracture site, a C-arm rotated by $15^{\circ}$ was used to check the reduction of the tibial plateau in anteroposterior and lateral views. Then, during the repair of the lateral meniscus, the articular surface at the fracture site was di-
Table 1. Demographic Data

\begin{tabular}{lc}
\hline \multicolumn{1}{c}{ Characteristic } & Data \\
\hline No. of patients & 25 \\
Sex & $17(68)$ \\
Male & $8(32)$ \\
Female & $49.28(22-68)$ \\
Age (yr), mean (range) & \\
Schatzker fracture type & 15 \\
II & 10 \\
III & \\
Cause of injury & $19(76)$ \\
Traffic accident & $3(12)$ \\
Slip down & $2(8)$ \\
Sports injury & $1(4)$ \\
Fall down & \\
Associated injury & $16(64)$ \\
Lateral meniscus tear & $6(24)$ \\
Medial collateral injury & $4(16)$ \\
ACL avulsion fracture & $4(16)$ \\
PCL injury & $1(4)$ \\
Medial meniscus tear &
\end{tabular}

Values are presented as number (\%).

ACL: anterior cruciate ligament, PCL: posterior cruciate ligament.

rectly visualized with arthroscopy.

For patients with large bone defects or osteoporosis that could disrupt maintenance of reduction, bone grafts were used: an allogeneic bone graft in 11 patients and an autologous bone graft obtained from the iliac crest in 2 patients.

Following the fracture treatment, arthroscopic repair of the lateral meniscus was performed. Transvers and longitudinal tears in the anterior and central portions of the meniscus were 
sutured with a modified all-inside repair technique using an 18-gauge spinal needle, whereas the all-inside repair was performed through the posterolateral portal for posterior tears ${ }^{4}$. The procedure was performed using No.2 PDS (Ethicon, Somerville, NJ, USA) and knots were tied using the Samsung Medical Center sliding method. For lateral meniscus tears where suture repair was unfeasible or horizontal tears, partial meniscectomy was done (Fig. 2). Conservative treatment was prescribed for patients with medial collateral ligament injuries and those without joint instability in spite of ACL avulsion fractures or PCL injuries. For the medial meniscal tear, partial meniscectomy was performed based on the arthroscopic evidence of a horizontal tear.

\section{Postoperative Rehabilitation}

Patients who underwent surgical treatment were immobilized in a long leg cast for 1 week. Isometric quadriceps exercises were initiated from the 1st postoperative day. Range of motion (ROM) exercises were gradually carried out in the brace from the 7th postoperative day to obtain $90^{\circ}$ of flexion/extension by $2-4$ weeks after surgery. Partial weight bearing was permitted when $90^{\circ}$ flexion was achieved. Full weight bearing was allowed from the 8 th postoperative week.

\section{Second-Look Arthroscopy and Metal Removal}

Bony union was observed at the last follow-up in 18 patients who had been surgically treated prior to June 2015. For these patients, metal removal and diagnostic second-look arthroscopy were performed. Metal removal was done at a mean of 14.8 months (range, 12 to 27 months) after surgery. In 11 patients with concomitant lateral meniscal injuries, diagnostic second-look arthroscopy was performed to assess any changes in the meniscus.

\section{Results}

Intraoperative $\mathrm{C}$-arm images and postoperative plain radiographs of the anteroposterior and lateral views of the tibial plateau revealed anatomical reduction of the fracture $(<1 \mathrm{~mm}$ articular step-off). The fracture was found united in all knees at
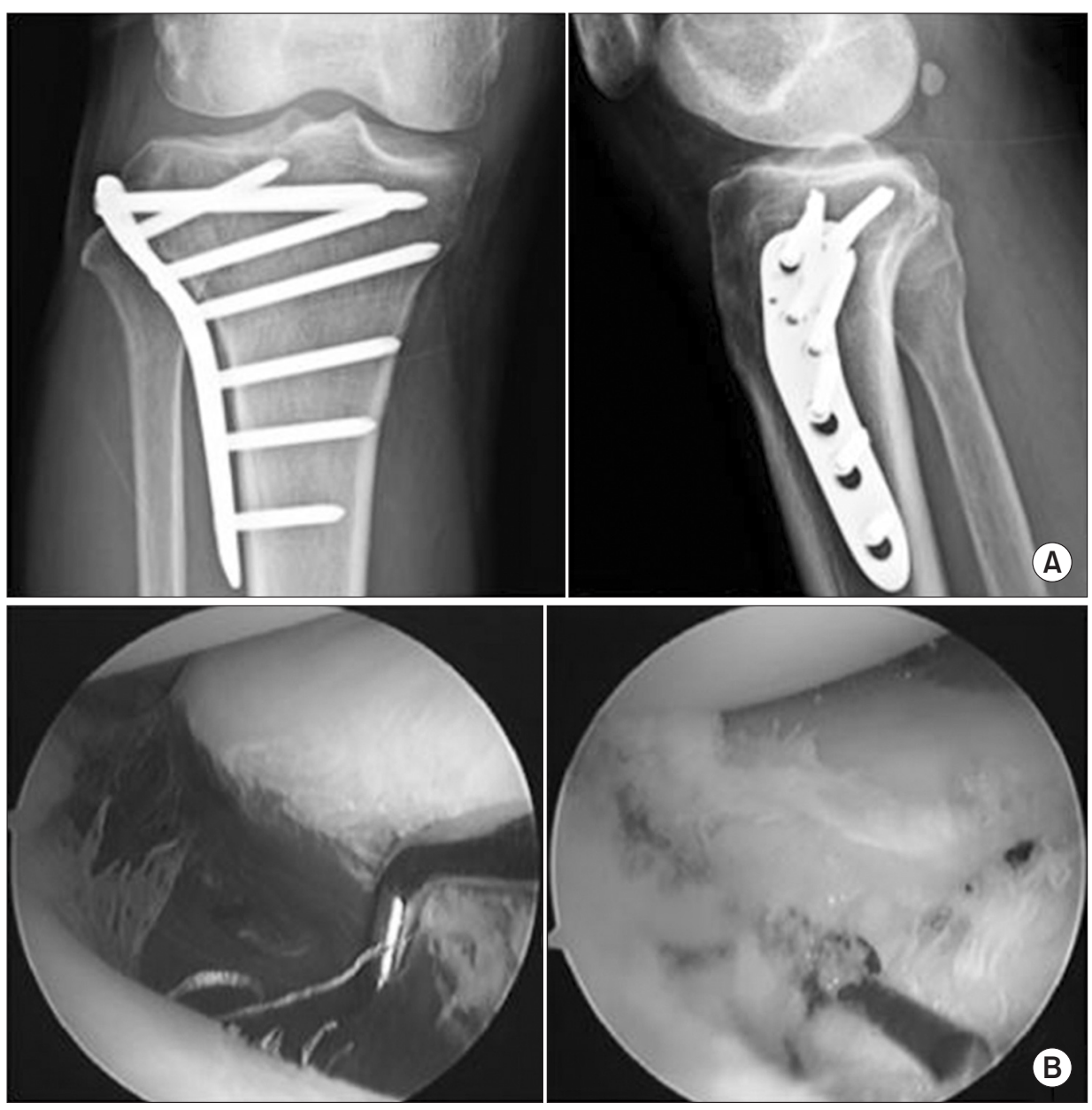

Fig. 2. (A) Postoperative anteroposterior and lateral radiographs. (B) Arthroscopic image of a lateral meniscal tear after repair. 
the last follow-up. In 2 patients, brisement under anesthesia was required due to restrictions on the ROM. There was no case of nonunion or delayed union necessitating a revision surgery. Infection or loss of reduction did not occur in all knees.

The concomitant lateral meniscal tears were present in 16 of the 25 patients with tibial plateau fractures. Longitudinal tears were the most common $(n=8)$, followed by radial tears $(n=3)$, horizontal tears $(n=3)$, and flap tears $(n=2)$ (Table 2$)$.

Of the 16 patients with lateral meniscal tears, suture repair was conducted in 10 and partial meniscectomy was performed in 2 because suture repair was not feasible in them. In the remaining 4 patients, additional procedure was not considered necessary because the extent of damage was small or minor.

During metal removal, a second-look arthroscopy was performed in 11 patients with lateral meniscal tears, which showed complete healing of the lateral meniscus in all knees (Fig. 3).

Four patients were not treated surgically although lateral meniscal tears were suspected based on MRI and arthroscopic examinations. One of these patients complained of persistent pain in spite of achievement of bony union at the last followup. The presence of a lateral meniscal tear was suspected based on follow-up MRI. Thus, arthroscopic examination was done to

Table 2. Lateral Meniscus Tear Type

\begin{tabular}{ll}
\hline Tear type & No. (\%) \\
\hline Horizontal & $3(18.7)$ \\
Longitudinal & $8(50)$ \\
Radial & $3(18.7)$ \\
Flap & $2(12.5)$ \\
\hline
\end{tabular}

confirm the diagnosis. Partial meniscectomy was performed for treatment, which resulted in pain relief in the patient.

\section{Case Report 1}

A 48-year-old female patient sustained a Schatzker type III lateral tibial plateau fracture in a pedestrian traffic accident. Concomitant injuries to the lateral meniscus (Fig. 4A) and medial collateral ligament were observed. The fracture was treated with open reduction and internal fixation. For the longitudinal meniscal tear, all-inside repair ${ }^{4)}$ using a spinal needle was performed. The patient was ordered to wear a brace for the medial collateral ligament injury. At 1 year after surgery, second-look arthroscopy and metal removal were performed, which showed complete healing of the meniscus (Fig. 4B).

\section{Case Report 2}

A 60-year-old male patient sustained a Schatzker type II lateral tibial plateau fracture in a vehicle accident. Although a lateral meniscal injury was present, the patient underwent only open reduction and internal fixation (Fig. 5A) for the fracture. There was no procedure performed for the meniscal tear.

At 1 year after surgery, stable bony union was obtained at the fracture site (Fig. 5B). However, he complained of persistent knee pain. Follow-up MRI confirmed the presence of a lateral meniscal tear (Fig. 5C). At the time of metal removal, a secondlook arthroscopy was performed. After partial meniscectomy, the symptom was relieved (Fig. 5D).
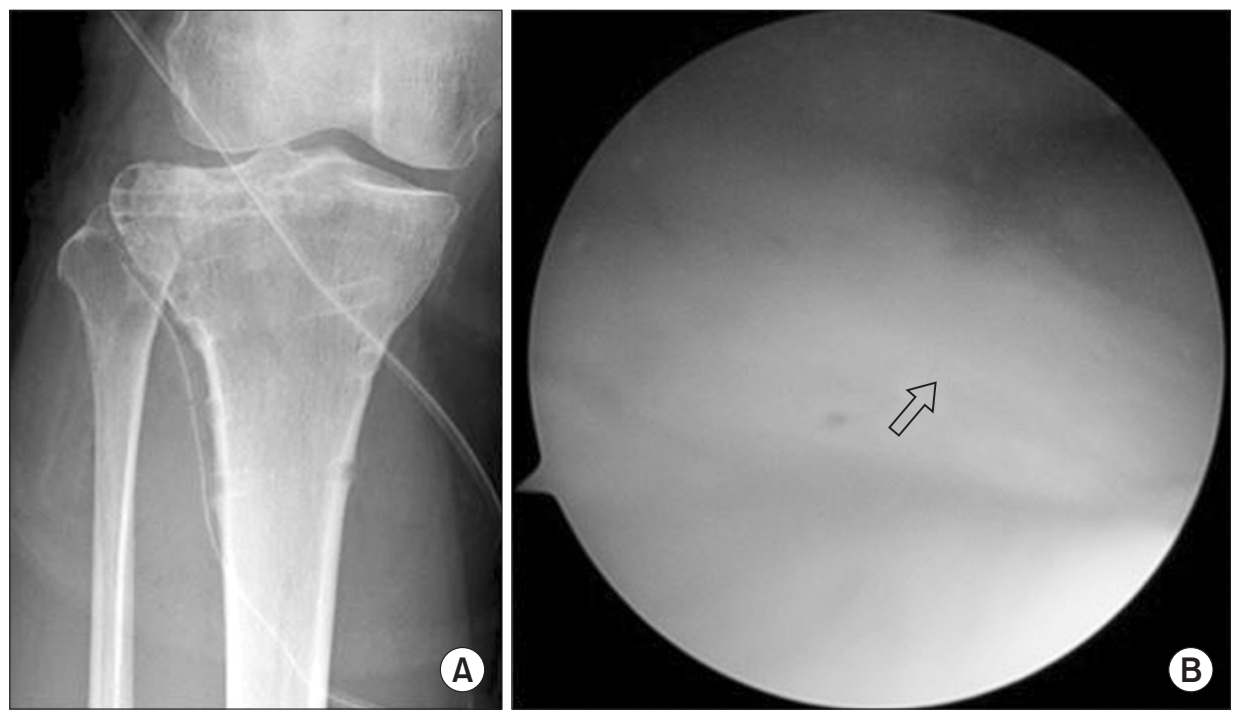

Fig. 3. (A) Post implant removal state. (B) Healed lateral meniscus (black open arrow: previous tear site). 

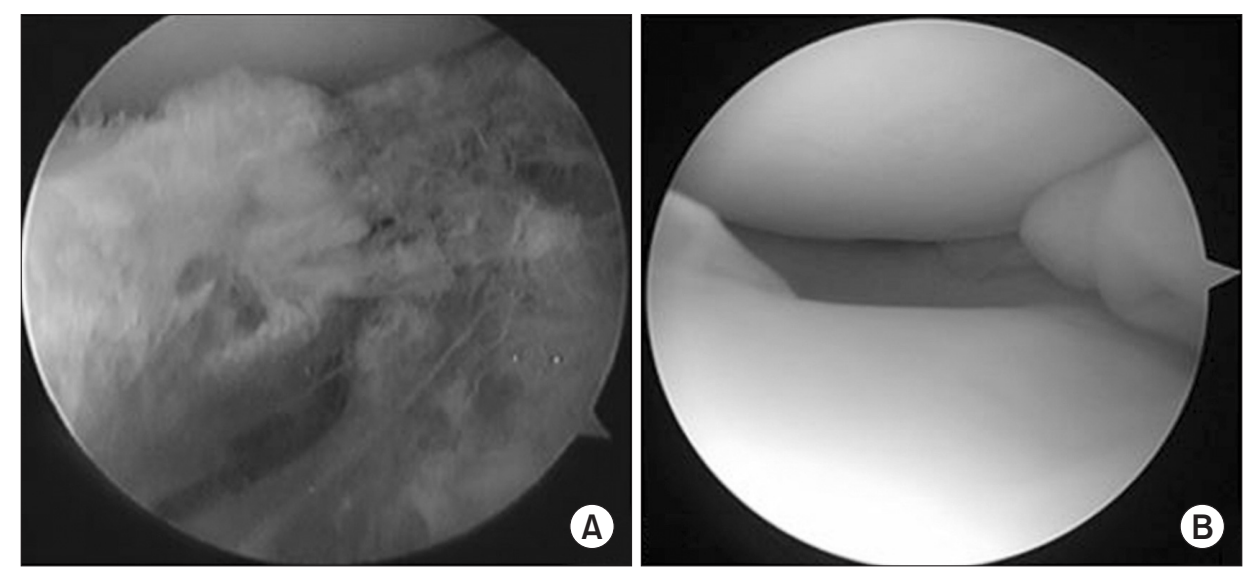

Fig. 4. Case report 1 (case no. 4). (A) Accompanied longitudinal tear of lateral meniscus. (B) Complete healing of lateral meniscus at second-look arthroscopy.
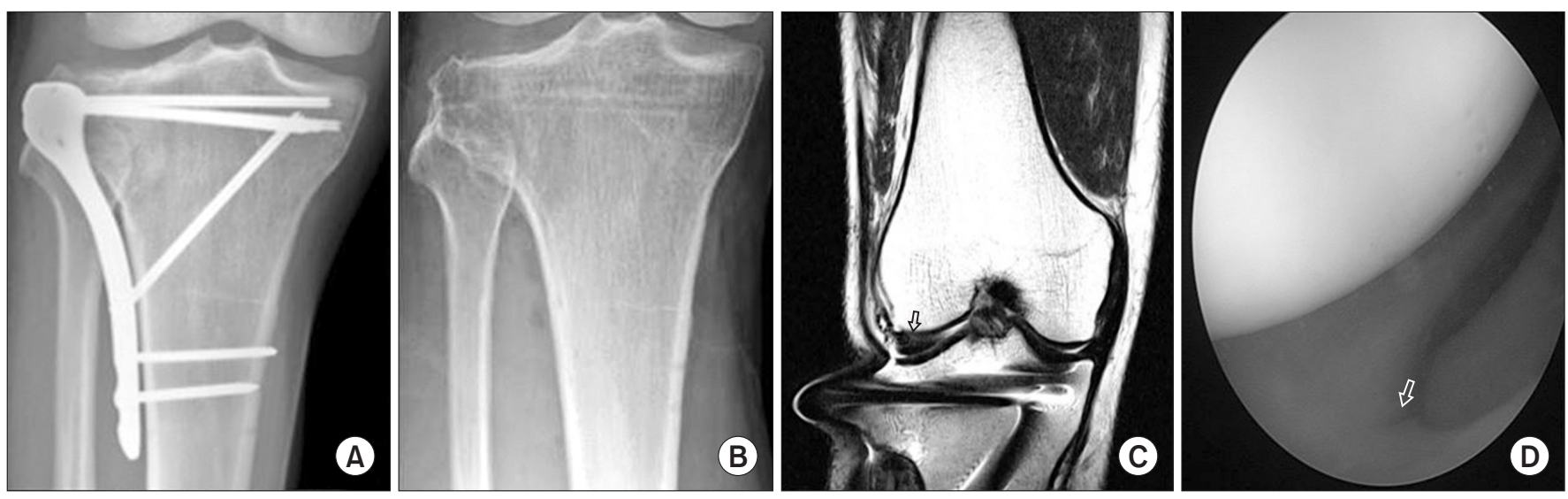

Fig. 5. Case report 2. (A) The immediate postoperative radiograph showing fixation by lateral plating. (B) Stable bony union. (C) Postoperative magnetic resonance imaging showing the healed tear site (black open arrow). (D) Second-look arthroscopy of the healed tear site (white open arrow).

\section{Discussion}

Tibial plateau factures are known to occur with meniscal tears in $36 \%$ to $61 \%$ of the cases ${ }^{5-9}$. In the current study, concurrent lateral meniscal tears were observed in $64 \%$ of patients with Schatzker type II or III fractures. Interestingly, it is common for surgeons not to report concomitant meniscal lesions in tibial plateau fractures treated without arthroscopy, indicating that meniscal tears may remain untreated.

With improved understanding and treatment of meniscal tears in the field of orthopedic surgery, it has been well established that the appropriate use of meniscal repair or meniscectomy for preservation of the meniscus would contribute to prevention of cartilage loss.

The incidence of posttraumatic osteoarthritis of the knee ranges from $17 \%$ to $28.9 \%^{10-13)}$, and predisposing factors include articular congruity, joint stability, meniscal injury, lower limb alignment, and patient's age $e^{14)}$.

The most important factor of those listed above is articular congruity, loss of which is termed as a step-off ${ }^{15)}$. The presence of lesions in the meniscus has also been associated with posttraumatic osteoarthritis in the long term ${ }^{16}$.

In the current study, the second-look arthroscopy revealed more satisfactory results in the knees where meniscal injuries, if present, were treated simultaneously at the time of reduction of lateral tibial plateau fractures. In particular, complete healing at the repair site was noted in knees where the lateral meniscus had been repaired.

In our opinion, the high success rate of meniscal repair in patients with combined lateral tibial plateau fractures can be attributed to the following three factors. First, meniscal repair was performed within the first week of injury. The meniscus was repaired during the surgery for the combined fracture, which was performed within 1 week of injury. Therefore, repair of an acute tear is expected to have a positive impact on healing. Second, the concurrent fracture reduction procedure facilitated bone marrow stimulation as in ACL reconstruction. Some studies have shown that $\mathrm{ACL}$ reconstruction combined with meniscal repair resulted 
in improved healing of the meniscus when compared to the result of meniscal repair alone ${ }^{17,18)}$. Therefore, simultaneous meniscal repair and fracture reduction can be considered to contribute to enhanced meniscal healing. Third, restrictions on postoperative ROM were conducive to the desired environment for healing of the repaired meniscus. Unlike the standard rehabilitation protocol prescribed after meniscal repair only, flexion was limited to $90^{\circ}$ and weight bearing was progressively increased over the period of 8 postoperative weeks to stabilize reduction of the fracture, which also contributed to enhanced stability at the repair site.

Currently, computed tomography is a standard imaging modality for the treatment of lateral tibial plateau fractures, whereas there are no guidelines on the use of MRI. However, it is our understanding that early MRI scanning would be helpful considering that the incidence of injuries to the meniscus and ligaments is high in patients with lateral tibial plateau fractures. In addition, it can be difficult to assess lesions on postoperative MRI scans due to the presence of internal fixation apparatus for fracture treatment.

The 60-year-old male patient case demonstrated that lateral meniscal tears can be satisfactorily treated and these lesions can be responsible for persistent joint pain, swelling, and effusion unless proper treatment is carried out.

Therefore, we suggest that 1) preoperative MRI should be an essential component of the management of lateral tibial plateau fractures and 2) treatment of a concomitant intraarticular lesion and fracture reduction should be performed simultaneously to improve clinical outcomes.

\section{Limitation}

One of the limitations of this study include the small sample size precluding statistical analysis. Statistical significance of our findings should be evaluated in further studies involving a larger study population.

Another limitation was the short follow-up period. With a longer follow-up, we could have demonstrated that the neglected meniscal lesions have an influence on the occurrence of posttraumatic osteoarthritis, resulting in relatively poor clinical outcomes.

Lastly, compared to randomized controlled trials, there was an increased probability of selection bias of this retrospective study. Since there was no control group for comparison, it was difficult to provide definitive results other than the clinical outcomes of repair of meniscal tears combined with lateral tibial plateau fractures.

\section{Conclusions}

Schatzker type II and III fractures of the lateral tibial plateau frequently occur with lateral meniscal damage. Internal fixation and meniscal repair resulted in excellent clinical outcomes. Therefore, we believe preoperative MRI should be performed to identify intraarticular lesions combined with lateral tibial plateau fractures. Simultaneous repair of meniscal injuries would reduce the risk of posttraumatic osteoarthritis, eventually improving long-term clinical outcomes.

\section{Conflict of Interest}

No potential conflict of interest relevant to this article was reported.

\section{References}

1. Bennett WF, Browner B. Tibial plateau fractures: a study of associated soft tissue injuries. J Orthop Trauma. 1994;8:1838.

2. Stahl D, Serrano-Riera R, Collin K, Griffing R, Defenbaugh B, Sagi HC. Operatively treated meniscal tears associated with tibial plateau fractures: a report on 661 patients. J Orthop Trauma. 2015;29:322-4.

3. Tscherne H, Lobenhoffer P. Tibial plateau fractures: management and expected results. Clin Orthop Relat Res. 1993;(292):87-100.

4. Cho JH. Arthroscopic all-inside repair of anterior horn tears of the lateral meniscus using a spinal needle. Knee Surg Sports Traumatol Arthrosc. 2008;16:683-6.

5. Abdel-Hamid MZ, Chang CH, Chan YS, Lo YP, Huang JW, Hsu KY, Wang CJ. Arthroscopic evaluation of soft tissue injuries in tibial plateau fractures: retrospective analysis of 98 cases. Arthroscopy. 2006;22:669-75.

6. Chan YS, Chiu CH, Lo YP, Chen AC, Hsu KY, Wang CJ, Chen WJ. Arthroscopy-assisted surgery for tibial plateau fractures: 2- to 10-year follow-up results. Arthroscopy. 2008;24:760-8.

7. Gill TJ, Moezzi DM, Oates KM, Sterett WI. Arthroscopic reduction and internal fixation of tibial plateau fractures in skiing. Clin Orthop Relat Res. 2001;(383):243-9.

8. Kode L, Lieberman JM, Motta AO, Wilber JH, Vasen A, Yagan R. Evaluation of tibial plateau fractures: efficacy of MR imaging compared with CT. AJR Am J Roentgenol. 1994;163:141-7. 
9. Mustonen AO, Koivikko MP, Lindahl J, Koskinen SK. MRI of acute meniscal injury associated with tibial plateau fractures: prevalence, type, and location. AJR Am J Roentgenol. 2008;191:1002-9.

10. Honkonen SE. Degenerative arthritis after tibial plateau fractures. J Orthop Trauma. 1995;9:273-7.

11. Lachiewicz PF, Funcik T. Factors influencing the results of open reduction and internal fixation of tibial plateau fractures. Clin Orthop Relat Res. 1990;(259):210-5.

12. Scheerlinck T, Ng CS, Handelberg F, Casteleyn PP. Mediumterm results of percutaneous, arthroscopically-assisted osteosynthesis of fractures of the tibial plateau. J Bone Joint Surg Br. 1998;80:959-64.

13. Mehin R, O'Brien P, Broekhuyse H, Blachut P, Guy P. Endstage arthritis following tibia plateau fractures: average 10year follow-up. Can J Surg. 2012;55:87-94.

14. Marsh JL, Buckwalter J, Gelberman R, Dirschl D, Olson S, Brown T, Llinias A. Articular fractures: does an anatomic reduction really change the result? J Bone Joint Surg Am. 2002;84:1259-71.

15. Giannoudis PV, Tzioupis C, Papathanassopoulos A, Obakponovwe O, Roberts C. Articular step-off and risk of posttraumatic osteoarthritis: evidence today. Injury. 2010;41:98695.

16. Hunter DJ, Zhang YQ, Niu JB, Tu X, Amin S, Clancy M, Guermazi A, Grigorian M, Gale D, Felson DT. The association of meniscal pathologic changes with cartilage loss in symptomatic knee osteoarthritis. Arthritis Rheum. 2006;54:795-801.

17. Horibe S, Shino K, Nakata K, Maeda A, Nakamura N, Matsumoto N. Second-look arthroscopy after meniscal repair: review of 132 menisci repaired by an arthroscopic inside-out technique. J Bone Joint Surg Br. 1995;77:245-9.

18. Tenuta JJ, Arciero RA. Arthroscopic evaluation of meniscal repairs: factors that effect healing. Am J Sports Med. 1994;22:797-802. 